\title{
Peak plantar pressure analysis using customized tactile sensory feedback system — A short report
}

\author{
M. Sathish Paul ${ }^{\mathrm{a}} \&$ B. Siva ${ }^{\mathrm{a}}$

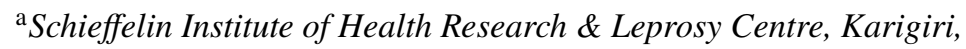 \\ Vellore, Tamil Nadu, India
}

Submitted 2 February 2021; Accepted 15 February 2021

\begin{abstract}
Summary Sensory neuropathy is prevalent in people affected by leprosy and diabetes. If neuropathy is left untreated it may lead to further impairments, which may be irreversible. Customized methods to identify and prevent further impairments in patients with sensory neuropathy are essential. The possibility of a sensory feedback system to identify pressure values on an anaesthetic foot was tested in this study. The feedback system to measure the peak plantar pressures was designed by integrating an electronic circuit to the data acquisition system. The sensor head of the Force Sensing Resistor (FSR's) placed at various plantar pressure areas of the foot, determined the respective peak plantar pressures, based on sensing force. Feedback about the peak plantar pressure through the alerting terminals helps the user alter his gait and maintain the pressure within a set threshold limit. The system could also help the health staff to determine the patient's peak plantar pressure and thus design customized therapeutic footwear.
\end{abstract}

Keywords: Sensory neuropathy, plantar pressure, force sensitive resistors, leprosy, diabetes

\section{Introduction}

Plantar neuropathic ulcers are those which result from sensory neuropathy, occurring predominantly in diabetic and leprosy affected patients. ${ }^{1,2}$ Interventions like debridement of necrotic tissues and wound dressing are often expensive for people to afford. ${ }^{3}$ Efforts on early identification of foot impairments are vital in any health care programme and could save the cost involved in managing plantar ulcers. With the loss of sensory inputs to patients with anaesthetic feet in leprosy and diabetes, it is vital for the patients to understand the varying peak plantar pressures. $^{4,5}$ Force Sensitive Resistors (FSR) are widely used in varied applications because of their simple working principle, low cost and accuracy in the results when interfaced with other wearable devices. ${ }^{6,7}$ The sensor's flexibility, weight and ultra thin properties make it a preferred tool in assessing plantar and palmar pressures. The resistance of the FSR varies

Correspondence to: M. Sathish Paul, Schieffelin Institute of Health Research \& Leprosy Centre, Karigiri, Vellore, Tamil Nadu, India (e-mail: sathishpau177@gmail.com) 


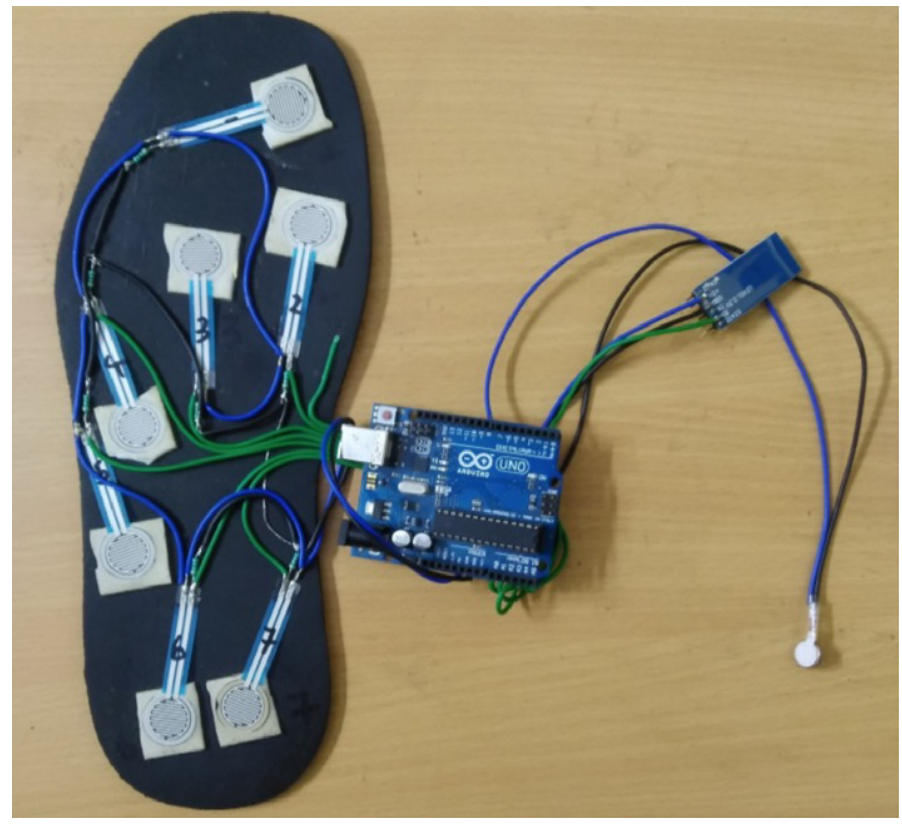

Figure 1 The entire setup of the plantar pressure feedback system operated by the integration of various FSR's , Arduino UNO and vibrating motor.

from $1 \mathrm{M} \Omega$ to about $10 \mathrm{k} \Omega$ for a load of $100 \mathrm{~g}$ to $10,000 \mathrm{~g} .{ }^{8,9}$ These specifications are ideal to analyse the pressure acting on the pressure heads (sensing area) of the sensor and transmitting it to the data acquisition system. The general working principle of force sensitive resistors is based on variation of resistance. The relationship between the output resistance and applied force are inversely proportional, resistance of the sensor is high when there is less or no force and varies when the force acts on it again. This study aims to test the efficacy of the data acquisition system that would identify and provide feedback of the peak pressure exerted on the identified plantar pressure points. With the pressure value actuating the alerting devices in the system, the patient could themselves alter and learn the ideal walking pattern within the threshold peak pressures.

\section{Short report}

Plantar pressure prone areas of the foot such as the Great toe (GT), 1st Metatarsal head (MT1), 5th Metatarsal Head (MT5), Lateral Border (LB) and the Heel (H) were identified in the patient. The sensory feedback system through the FSR's was designed to provide feedback on the pressure variations. Integrated circuits and electronic devices were used for transferring the data from the sensor to microcontroller triggers in the alerting device. The threshold pressure value for triggering the alert is controlled by Arduino IDE (Integrated Development Environment) software. A Micro Cellular Rubber (MCR) insole was trimmed and plantar pressure prone areas identified by the Harrison mat were marked for the placement of FSR (Figure 1). FSR's were then connected to the Arduino UNO which acts as a data acquisition board. ${ }^{10,11}$ Arduino UNO used in the data acquisition system is an open source microcontroller with a feature of compatible programmability. The integrated Bluetooth module (HC-05) 


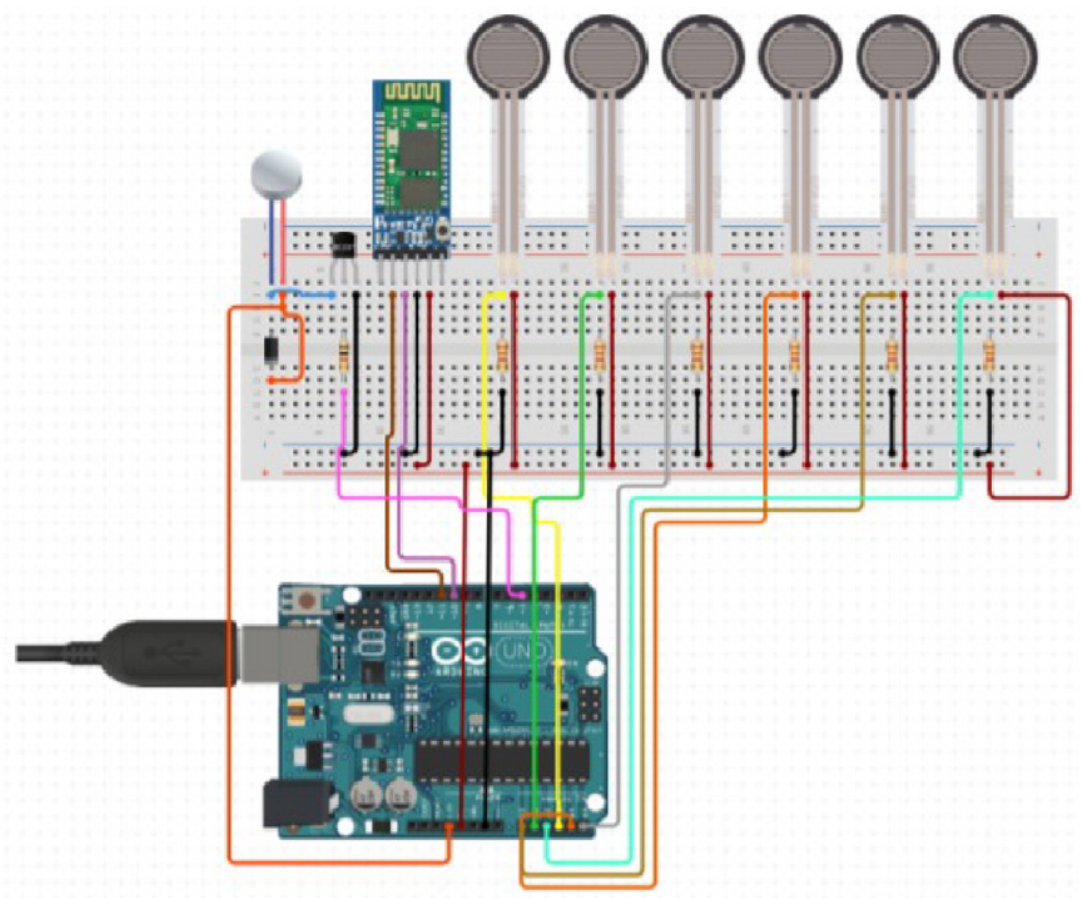

Figure 2 An experimental setup showing the actuation of vibrating motor in relation to the force applied on the FSR's.

sends the data to the computer as well as actuating the coin vibration motor (Figure2). The Bluetooth module works with the input of 3.3-6 V and $30 \mathrm{~mA}$ current.

The FSR heads were placed in the identified plantar pressure regions to obtain accurate pressure values. Respective force values exerted on the FSR heads placed at the plantar pressure area of the foot were obtained through the serial monitor. A $10 \mathrm{~V}$ battery was used to power this circuit. All Force sensitive resistors and the circuit board were adequately soldered to minimize the error caused by misplacement of circuits. After the integration of the circuit, the FSR heads were placed at the plantar pressure prone areas of the foot identified on the insole. Then the insole with the sensor was fixed into the patient's shoes to obtain the values of forces acting on the plantar region of the foot while walking (Figure3). The data acquired were evaluated to recognize the peak pressure on the plantar area of the foot which could help in determining the threshold limit for integrating an alerting circuit.

The coin vibrator is connected to the pin of Arduino which acts as the alerting terminal. The vibration depends on the force applied on force sensitive resistor head of the FSR's. This specialized insole would trigger the vibrator when the force on the sensors exceeds the threshold limit. The threshold limit for the patient was identified based on the repeated walking pattern of the patient with the insoles. The sensory feedback system was provided to the patient and the pressure variations on doing his activities of daily living (ADL) were recorded and monitored through the data acquisition system (Figure4).

This prototype was developed based on above technical details and Arduino board was coded by considering the patient weight. 


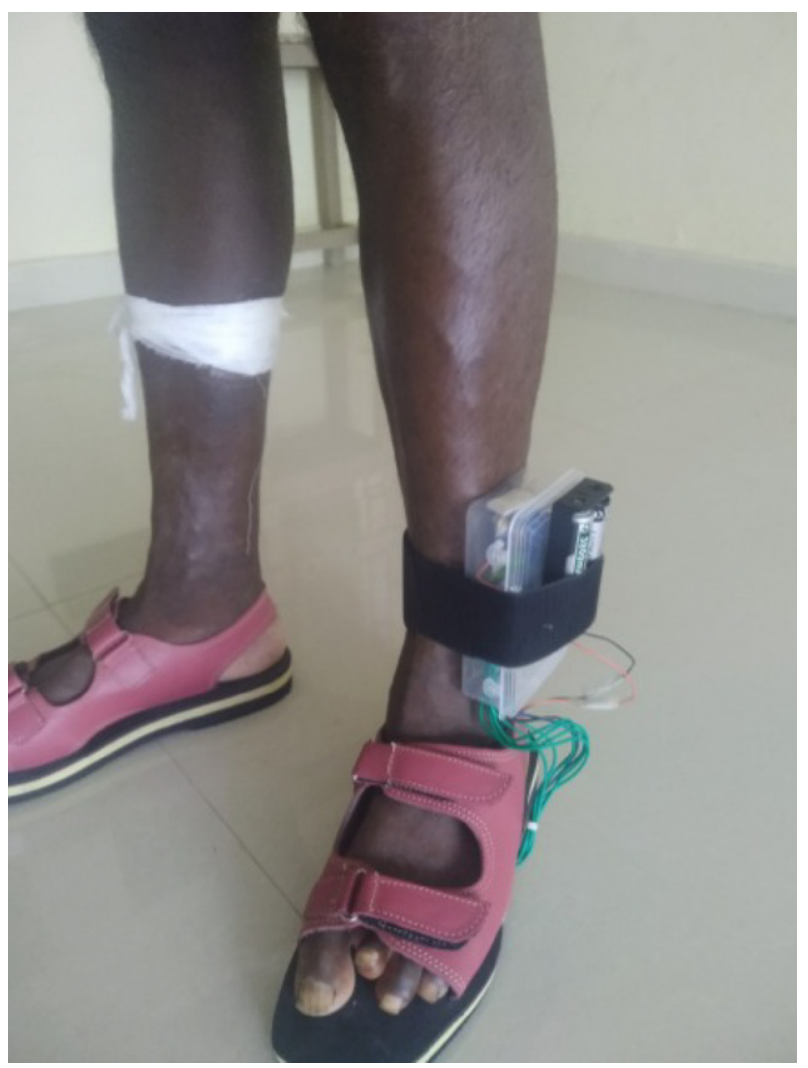

Figure 3 The prototype of tactile sensory feedback system installed on a patient with anaesthetic feet.

\section{Discussion}

The patient using the sensory feedback system was alerted when the peak plantar pressures exceeded the threshold values. The vibrator integrated into the data acquisition system alerted the patient when the plantar pressure threshold value was exceeded. The feedback helps the patient alter their gait. Suitable sensors were identified based on the ease of integration, sensing product size and the efficiency of power. The FSR's which were used in the study are cost effective and were readily available with the local electronic vendors. The cost of the single feedback system unit developed was below $\$ 150$, which could be cheaper if manufactured in bulk. Different sensors like load cells, strain gauges, and piezo resistive sensors are being used and are available in the market for identifying plantar pressure. Though there are many methods for identifying plantar pressure, this experimental setup establishes the ease of identifying peak pressure and providing biofeedback to the user through FSR's. Early recognition of plantar pressure, timely management, altering life styles and customized therapeutic innovations for high risk individuals could prevent foot ulcers, protect limbs and effectively save lives. ${ }^{12,13}$ The biofeedback would help the user to alter and adapt to a gait pattern that would help in preventing plantar ulcers. Recent development in the Internet of Things (IoT) technology facilitates the effective use of sensors and wireless modules to analyse and to transfer data from the device to the computer. ${ }^{14,15}$ 


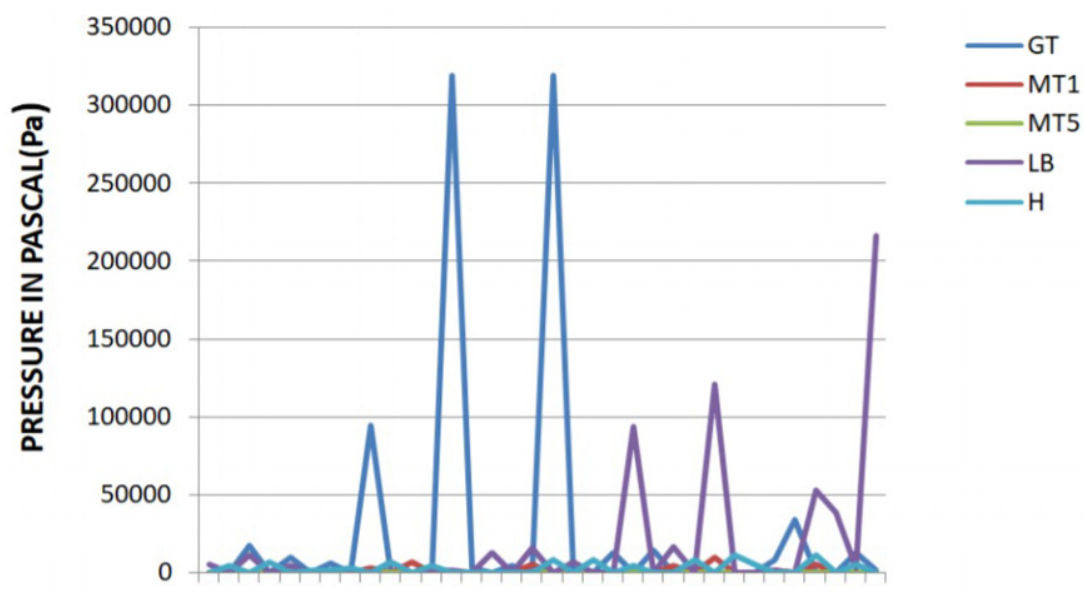

$\begin{array}{lllllllllllllllll}1 & 3 & 5 & 7 & 9 & 11 & 13 & 15 & 17 & 19 & 21 & 23 & 25 & 27 & 29 & 31 & 33\end{array}$

NUMBER OF STEPS

Figure 4 The graphical representation of peak plantar pressures identified at the plantar areas of the foot on each steps during gait.

\section{Conclusion}

A cost effective tactile sensory feedback system could help in identifying and preventing peak plantar pressures. We have presented here the design of such a system. The efficacy of the feedback system needs to further studied through a clinical trial with a cohort of patients.

\section{Conflict of interest}

Authors declare no conflict of interest.

\section{Funder}

American Leprosy Mission (ALM).

\section{Funding statement}

The authors would like to acknowledge the American Leprosy Missions (ALM) for funding this research work. The research work received funding through the project, funding number 4384.

\section{Patients consent statement}

Consent was taken while recruiting the patient for the study.

\section{Acknowledgement}

The authors would like to acknowledge Dr. Jerry Joshua, Director, SIHRLC Karigiri for his valuable inputs and suggestions. 


\section{References}

1 Ndip A, Ebah L, Mbako A. Neuropathic diabetic foot ulcers - evidence-to-practice. Int J Gen Med, 2012; 5: 129-134, doi:10.2147/IJGM.S10328.

2 Del Core MA, Ahn J, Lewis RB, Raspovic KM, Lalli TAJ, Wukich DK. The evaluation and treatment of diabetic foot ulcers and diabetic foot infections. Foot Ankle Orthop, 2018; doi:10.1177/2473011418788864.

3 Tallis A, Motley TA, Wunderlich RP et al. Clinical and economic assessment of diabetic foot ulcer debridement with collagenase: results of a randomized controlled study. Clin Ther, 2013; 35(11): 1805-1820, doi:10.1016/j .clinthera.2013.09.013.

4 Paul MSK, Kumar DP, Govindasamy K. Physical rehabilitation in leprosy. In: Scollard DM, Gillis TP (eds), International Textbook of Leprosy. American Leprosy Missions, 2019, posting date.

5 Paisey RB, Darby T, George AM et al. Prediction of protective sensory loss, neuropathy and foot ulceration in type 2 diabetes. BMJ Open Diabetes Res Care, 2016; 4(1): e000163. Published 2016 May 5.

6 Giovanelli D, Farella E. Force sensing resistor and evaluation of technology for wearable body pressure sensing. J Sens, 2016; 1-13.

7 Park JS, Lee CM, Koo S, Kim CH. Gait phase detection using force sensing resistors. IEEE Sens J, 2020; 20(12): 6516-6523.

8 Sadun A. S., Jalani J.. Sukor J. A.Force Sensing Resistor (FSR): a brief overview and the low-cost sensor for active compliance control. In: First International Workshop on Pattern Recognition, 2016, vol. 10011. doi: $10.1117 / 12.2242950$.

9 Hollinger A, Wanderley M. Evaluation of commercial force-sensing resistors. In: Proceedings of the International Conference on New Interfaces for Musical Expression, 2006.

10 Issa AH, Thuneibat S. Sensors application using PIC16F877A microcontroller. Am J Remote Sens, 2016; 4(3): 13-18.

11 Chiu CH. Microcontroller-based feedback control laboratory experiments. IJEP, 2014; 4(3): 60-66.

12 Paul S, Vijayakumar R, Mathew L. Finite element model-based evaluation of tissue stress variations to fabricate corrective orthosis in feet with neutral subtalar joint. Prosthet Orthot Int, 2017; 41(2): 157-163.

13 Sathish KP, Rekha V, Sudesh S. Customized Insole fabrication for foot deformities in Leprosy patients. $J$ Med Devices, 2014; 8(2): 020950.

14 Ghaida HA, Mottet S, Goujon JM. Foot modeling and smart plantar pressure reconstruction from three sensors. Open Biomed Eng J, 2014; 8: 84-92.

15 Sorrentino I, Andrade Chavez FG, Latella C. A novel sensorised insole for sensing feet pressure distributions. Sensors, 2020; 20(3): 747. 